\title{
STUDY OF MEDICAL MANAGEMENT OF ECTOPIC PREGNANCIES
}

Yamini Patil1 ${ }^{1}$ Nikunj Sabhaya ${ }^{2}$

${ }_{1}$ Associate Professor, Department of Obstetrics and Gynaecology, Krishna Institute of Medical Sciences Deemed University, Karad. 2Junior Resident, Department of Obstetrics and Gynaecology, Krishna Institute of Medical Sciences Deemed University, Karad.

\section{ABSTRACT}

\section{BACKGROUND}

Ectopic pregnancy is a significant cause of morbidity and mortality in the first trimester of pregnancy. Treatment with Methotrexate (MTX), an antimetabolite chemotherapeutic agent that binds to the enzyme dihydrofolate reductase, which is involved in the synthesis of purine nucleotides. This interferes with DNA synthesis and disrupts cell multiplications, achieves results comparable to surgery for treatment of appropriately selected ectopic pregnancies. Surgery is specifically indicated in ruptured ectopic and when MTX is contraindicated.

Aim- To evaluate the medical management of Ectopic pregnancy in tertiary care hospital.

\section{MATERIALS AND METHODS}

Prospective study carried out on patients who came with unruptured ectopic pregnancies at our hospital within 2-year period of study.

\section{RESULTS}

- Failure rate of MTX therapy at Krishna Hospital is 8.33\%.

- $\quad$ Success rate of MTX therapy at Krishna Hospital is $91.70 \%$.

\section{CONCLUSION}

- $\quad$ Both medical therapy or conservative surgery should be viewed as appropriate first line therapies in many early unruptured ectopic pregnancies.

- $\quad$ Multiple dose MTX treatment has a lower failure rate than single dose MTX. Single dose MTX is sufficient to treat persistent trophoblastic tissue after salpingostomy and ectopic pregnancies associated with low initial HCG values.

- Postoperative, prophylactic, single dose systemic MTX may reduce the incidence of persistent ectopic pregnancy after salpingostomy.

Medical therapy appears more cost-effective than surgery except when the initial HCG level is high and/or embryonic cardiac activity is observed.

\section{KEYWORDS}

Ectopic Pregnancy.

HOW TO CITE THIS ARTICLE: Patil Y, Sabhaya N. Study of medical management of ectopic pregnancies. J. Evolution Med. Dent. Sci. 2017;6(25):2073-2077, DOI: 10.14260/Jemds/2017/451

\section{BACKGROUND}

Ectopic pregnancy is a significant cause of maternal morbidity and mortality, especially in India. While mortality from ectopic pregnancy has been on the decrease in the developed countries, despite the increase in the incidence it is not so in the developing countries, where it is the major cause of maternal mortality.

More than $95 \%$ of ectopic pregnancies occur in the fallopian tube. The other sites include ovaries, cervix, caesarean scar, broad ligament and abdominal cavity. Combined intrauterine and extrauterine pregnancy (Heterotopic pregnancy) though are rare in spontaneous pregnancies ( 1 in 3000 - 4000), has been recorded in up to $3 \%$ of pregnancies from assisted reproduction. ${ }^{1}$

Financial or Other, Competing Interest: None.

Submission 02-02-2017, Peer Review 16-03-2017,

Acceptance 22-03-2017, Published 27-03-2017.

Corresponding Author:

Dr. Yamini Patil,

\#450, Maher Hospital,

Shaniwar Peth, Karad-415110

Maharashtra.

E-mail: drnikunjsabhaya@gmail.com

DOI: $10.14260 /$ jemds $/ 2017 / 451$

\section{(c) $(1)$}

Several factors have been shown to increase the risk of ectopic pregnancy. These risk factors share a common mechanism of action- namely interference with the ciliary functions of fallopian tube. ${ }^{1}$ It has been observed that pelvic inflammatory disease is the most common risk factor for ectopic pregnancy and early treatment of the disease does not necessarily prevent tubal damage. The other reported aetiological factors include previous ectopic pregnancy, endometriosis, previous tubal surgery, infertility and infertility treatments.

Previous caesarean sections, tubal spasm, congenital defects of the fallopian and psychological and emotional factors have also been implicated.

In most cases, surgery is the mode of treatment. The surgical treatment may either be an open laparotomy or laparoscopic depending on the surgeon's skill, availability of equipment and clinical state of the patient. The management of ectopic pregnancy has been revolutionised over the past few decades. This has resulted in emergence of several nonsurgical options to what had once thought to be a solely surgically treatable condition. An earlier diagnosis can be made with transvaginal ultrasound and quantitative serum $\beta$ hCG. This increases the chances of success of medical 
treatment and minimises its morbidity, mortality and financial burden.

There have been some palpable changes in the diagnosis and treatment of ectopic pregnancy over the past decade at the study centre. This study therefore determines the incidence, associated risk factors, the currently available treatment modalities and suggests means of reducing the incidence and morbidity/mortality associated with ectopic pregnancy.

Ectopic pregnancy is a significant cause of morbidity and mortality in the first trimester of pregnancy. Currently high index of suspicion, serial hormone assays and transvaginal ultrasonography facilitates the diagnosis and treatment of ectopic pregnancy before rupture occurs. Early diagnosis and timely treatment have resulted in a dramatic decline in morbidity due to ectopic pregnancy.

Treatment with Methotrexate (MTX), an antimetabolite chemotherapeutic agent that binds to the enzyme dihydrofolate reductase, which is involved in the synthesis of purine nucleotides, this interferes with DNA synthesis and disrupts cell multiplications, achieves results comparable to surgery for treatment of appropriately selected ectopic pregnancies. In some protocols, folinic acid (leucovorin) is given to bypass the metabolic block induced by MTX and thus rescue the normal cells from toxicity. Many unruptured ectopic pregnancies can be managed with either surgery or methotrexate. Surgery is specifically indicated in ruptured ectopic and when MTX is contraindicated.

Treatment with MTX is an especially attractive option when the pregnancy is located on the cervix or ovary or in the interstitial or the cornual portion of the tube. Surgical treatment in these cases is always associated with risk of haemorrhage, hysterectomy and oophorectomy. ${ }^{2}$

\section{Aim}

To evaluate the medical management of Ectopic pregnancy in tertiary care hospital.

\section{Study}

Prospective study carried out in patients came with unruptured ectopic pregnancies at tertiary care centre in a period of 2-year study.

\section{MATERIALS AND METHODS}

This was prospective study with collection of all the cases of ectopic pregnancy managed at Krishna Hospital, Karad, from January 2015 to December 2016. All the cases of ectopic pregnancy in the Gynaecology ward were selected. The information on the age, parity, marital status, highest level of education and gestational age were obtained. The other information extracted included the risk factors and clinical presentations.

\section{Patient Selections}

- Haemodynamically stable women.

- Not persistent abdominal pain.

- Serum B-HCG level- $<5000 \mathrm{mIU} / \mathrm{L}$.

- Adnexal mass $<=3.5 \mathrm{~cm}$.

- Minimal free fluid in USG.

- Normal complete blood count and LFTs, Creatinine.

\section{Risks of Methotrexate Treatment ${ }^{3}$}

When administering methotrexate therapy, it is essential to remain aware of the risks and side effects of medical management. Rupture of ectopic pregnancy during methotrexate treatment ranges from $7 \%-14 \%$. The rate of increase in HCG is one predictor of rupture. In this particular study, 50 women were diagnosed with ectopic pregnancy and treated with methotrexate. A total of 47 women exhibited resolution of ectopic, while 3 posted for suction and evacuation. None of them ruptured. Most ruptured ectopic pregnancies occur when there is an increase of HCG, at least $66 \%$ after 48 hours persistently rising HCG concentration despite treatment.

Side effects should not be overlooked with methotrexate treatment. Most common side effects are mild and include nausea, vomiting, stomatitis, diarrhoea and elevated liver function tests. Rare, but severe side effects include nephrotoxicity, interstitial pneumonitis and alopecia dermatitis. Side effects can be limited by dose and length of treatment.

\section{Contraindications to Methotrexate}

\section{Absolute Contraindications}

- Intrauterine pregnancy.

- Evidence of immunodeficiency.

- Moderate-to-severe anaemia, leucopoenia or thrombocytopenia.

- Sensitivity to MTX.

- Active pulmonary disease.

- Active peptic ulcer disease.

- Clinically important hepatic and renal dysfunction.

- Breastfeeding.

- Ruptured ectopic pregnancy.

- Haemodynamically unstable patient.

\section{Relative Contraindications}

- High initial serum B-HCG level > 5000 mIU/L.

- Ectopic pregnancy >4 $\mathrm{cm}$ size by transvaginal sonography.

- Embryonic cardiac activity by TVS.

- Refusal to accept blood transfusion.

- Inability to participate in followup.

\section{Treatment Protocols for Ectopic Pregnancy ${ }^{4}$} Pre-Treatment Checks

- Discuss the options for treatmentexpectant/medical/surgical.

- $\quad$ CBC, RFTs, LFTs, $\beta$ HCG, Blood Group.

- Satisfy inclusion and exclusion criteria.

- Obtain written consent.

- Calculate the Patient Body Surface Area from height and weight.

- Prescribe methotrexate as per the dosage regimen.

\section{Single Dose Regimen}

- One dose of methotrexate on day $1 ; 50 \mathrm{mg} / \mathrm{m}^{2}$ body surface area.

- Serum B-HCG level day 1 (baseline), day 4 and day 7. 


\section{Indication for Additional Dose}

- If serum B-HCG level does not decline up to $15 \%$ from day 4 to day 7.

- Serum B-HCG post-therapy surveillance should be done until it becomes undetectable.

- At our hospital, 25 patients had received single-dose methotrexate therapy for ectopic pregnancy.

- Among them serum B-HCG level of 21 patients declined more than $15 \%$ on day 4 .

- Out of remaining 4 patients, 2 patients got decline in serum B-HCG level on day 7 and 2 patients with heterotopic pregnancy got posted for suction and evacuation.

\section{Multi-Dose Regimen}

- Four doses of methotrexate to be given until Serum BHCG level declines by $15 \%$.

- $\quad$ Dosage- $1 \mathrm{mg} / \mathrm{kg}$ on days $1,3,5,7$.

- It is associated with leucovorin on days 2, 4, 6, 8.

- Serum B-HCG level on day 1, 3, 5, 7.

Indication for Additional Dose

If serum B-HCG level declines less than 15\%, then give additional dose and repeat serum B-HCG level in $48 \mathrm{hrs}$. and compare with previous value. Maximum four doses are allowed.

Leucovorin which is folinic acid given with multidose regimen to buffer the side effects of MTX, which can lead to bone marrow depression also.

At our hospital, 25 patients had received multidose MTX therapy. Among them, 24 patients responded well to treatment.

One patient of 7 weeks of cervical live ectopic pregnancy posted for suction and evacuation after receiving 4 doses of MTX with leucovorin.

\section{Post-Treatment Management}

HCG- Weekly serial HCG followup needed until < 10 IU/L.

USG- There appears to be no clinical benefit from routine serial ultrasound examinations. After treatment, the ectopic pregnancy is often noted to increase in size and may persist for weeks on serial USS examinations. This could represent a haematoma rather than persistent trophoblastic tissue and is not predictive of treatment failure. However, USG evaluation for peritoneal free fluid is indicated for women with severe abdominal/pelvic pain.

\section{Advice the Woman To}

- Avoid vaginal intercourse until HCG is undetectable.

- Avoid pregnancy for three months due to the theoretical risk of teratogenicity with methotrexate.

- Avoid pelvic exams during surveillance of methotrexate therapy due to the risk of tubal rupture.

- Avoid sun exposure to limit risk of methotrexate dermatitis.

- Avoid foods and vitamins containing folic acid.

- Avoid non-steroidal anti-inflammatory drugs, as the interaction with methotrexate may cause bone marrow suppression, aplastic anaemia or gastrointestinal toxicity. Paracetamol with or without codeine is recommended for pain relief.

\begin{tabular}{|c|c|}
\hline Age (yrs.) & No. of Patients \\
\hline $18-24$ & 27 \\
\hline $25-30$ & 20 \\
\hline $31-35$ & 3 \\
\hline \multicolumn{2}{|c|}{ Table 1. Incidence of Ectopic Pregnancy } \\
in Relation with Maternal Age
\end{tabular}

Out of the 50 patients, 27 lie under the age group of 18 27 years, 20 lie under 25 - 30 years and 3 under 31 - 35 years.

\begin{tabular}{|c|c|}
\hline Parity & No. of Patients \\
\hline Primigravida & 25 \\
\hline Second gravida & 23 \\
\hline \multicolumn{2}{|c|}{ Multigravida } \\
\hline \multicolumn{2}{|c|}{ Table 2. Incidence of Ectopic Pregnancy } \\
in Relation with Parity \\
\hline
\end{tabular}

Out of the 50 patients, 25 were primigravida, 23 were second gravida and 2 were multigravida.

\begin{tabular}{|c|c|}
\hline Period of Gestation & Number \\
\hline 4 weeks - $\leq 5$ weeks & 8 \\
\hline 5 weeks $-\leq 6$ weeks & 18 \\
\hline 6 weeks $-\leq 7$ weeks & 18 \\
\hline 7 weeks - $\leq$ 6 weeks & 6 \\
\hline $\begin{array}{c}\text { Table 3. Incidence of Ectopic Pregnancies } \\
\text { in Relation with Period of Gestation }\end{array}$ \\
\hline
\end{tabular}

\begin{tabular}{|c|c|}
\hline Gestational Sac Size & Number \\
\hline No sac & 4 \\
\hline $1-2 \mathrm{~cm}$ size & 6 \\
\hline $2-2.5 \mathrm{~cm}$ size & 24 \\
\hline $2.5-3 \mathrm{~cm}$ size & 10 \\
\hline $3-3.5 \mathrm{~cm}$ size & 6 \\
\hline $\begin{array}{c}\text { Table 4. Incidence of Ectopic Pregnancies } \\
\text { in Relation with Gestational Sac Size }\end{array}$ \\
\hline
\end{tabular}

\section{Failure Rates of MTX Therapy}

At our hospital, 50 patients of unruptured ectopic pregnancy received MTX therapy. Among them 2 patients who received single dose MTX therapy and 1 patient who received multidose therapy were posted for suction and evacuation.

Failure rate of MTX therapy at our hospital is $6 \%$.

\begin{tabular}{|c|c|c|}
\hline $\begin{array}{c}\text { Serum B-HCG } \\
\text { Level (mIU/L) }\end{array}$ & $\begin{array}{c}\text { Failure Rate in Menon } \\
\text { Study (2007) }\end{array}$ & $\begin{array}{c}\text { Failure Rate in } \\
\text { Present Study }\end{array}$ \\
\hline$<1000$ & $1.5 \%$ & $1.3 \%$ \\
\hline $1000-2000$ & $5.6 \%$ & $5.7 \%$ \\
\hline $2000-5000$ & $3.8 \%$ & $4 \%$ \\
\hline $5000-10000$ & $14.3 \%$ & $13.6 \%$ \\
\hline \multicolumn{2}{|c|}{ Table 5. Comparison of Failure Rate with Serum } \\
B-HCG Level between Present Study and Menon Study \\
\hline
\end{tabular}

Adnexal foetal cardiac activity is present.

Size of the gestational mass $>4 \mathrm{~cm}$

High initial serum B-HCG level $>5000 \mathrm{mIU} / \mathrm{L}$

Presence of peritoneal blood.

Rapidly increasing serum B-HCG level > 50\%/48 hrs. before MTX therapy.

Continued rapid rise in serum B-HCG level during MTX therapy.

Table 6. Predictors of Failure of MTX Therapy 


\begin{tabular}{|c|c|c|c|}
\hline & Single Dose & Multi-Dose & Total \\
\hline $\begin{array}{l}\text { No. of patients who } \\
\text { received MTX }\end{array}$ & 25 & 25 & 50 \\
\hline $\begin{array}{l}\text { No. of patients not } \\
\text { responded to MTX }\end{array}$ & 2 & 1 & 3 \\
\hline Failure Rate & $8 \%$ & $4 \%$ & 6\% \\
\hline \multicolumn{4}{|c|}{ Table 7. Failure Rates of MTX Therapy } \\
\hline
\end{tabular}

\section{Success Rates of MTX Therapy}

- In present study, $92 \%$ success rate with single dose MTX when the ectopic mass was $<3.5 \mathrm{cms}$.

- Most studies report increased failure rates, if there is cardiac activity.

- Overall success rate of MTX therapy at our hospital is $94 \%$.

\section{Treatment Side Effects}

- These regimens are associated with minimal laboratory changes and symptoms.

- Most of the studies shows adverse effects were resolved within 4 - 5 days after MTX therapy.

- Most common side effect is abdominal pain, which is separation pain relieved by analgesics.

- Chances of Liver involvements are $12 \%$, Stomatitis are $6 \%$, Gastroenteritis are $1 \%$.

- There was no severe side effect noted among patients who received MTX therapy.

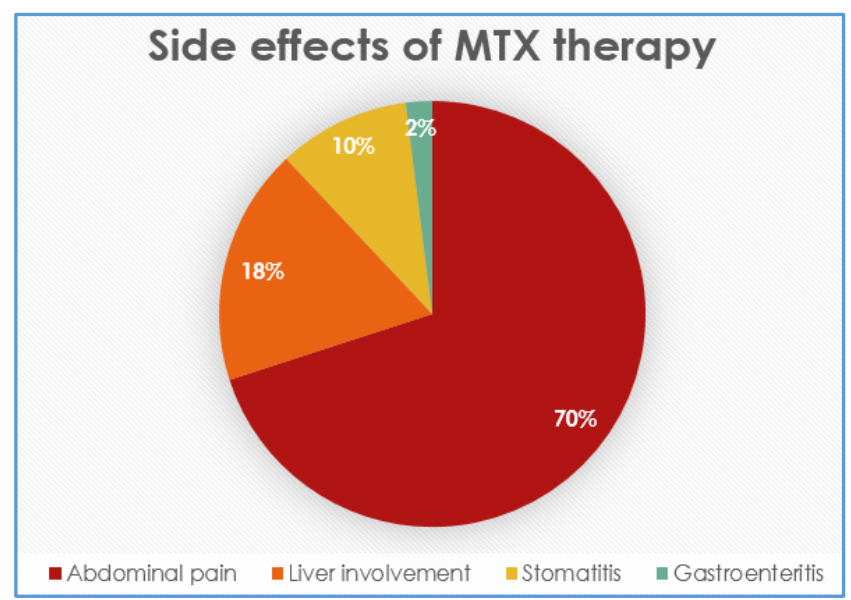

Graph 1. Side Effects of Methotrexate Therapy

\section{Monitoring Therapy Efficacy}

Serum B-HCG levels are used to monitor response to both medical and surgical therapy.

After single dose MTX therapy mean serum B-HCG level increase for the first 4 days and then gradually decline with a mean resolution time of 27 days.

With multidose therapy, serum B-HCG levels are measured at $48 \mathrm{hrs}$. intervals until levels are achieved.

Once the appropriately dropping of levels are achieved in either regimen, then serum B-HCG levels to be measured every weekly until it becomes undetectable.

\section{DISCUSSION}

Ectopic pregnancy is a major obstetric problem. The exact aetiology being unknown, it cannot be predicted or prevented. The present study was conducted to see the outcome of medical management of ectopic tubal gestation with Methotrexate. It was noted that out of 50 patients included, 94\% were successfully treated with intramuscular Methotrexate; 25 out of 50 were given single dose and $2(8 \%)$ were posted for suction and evacuation. The other 25 treated with multiple doses, 1 (4\%) required suction and evacuation; $88 \%$ of the patients were of the age 20 - 30 years; $50 \%$ of the cases were primigravida and other $50 \%$ were multigravida. This included those with previous abortions or ectopic pregnancy. Majority of the cases, i.e. 36 (72\%) were diagnosed at the gestational age of $5-7$ weeks. Early diagnosis is crucial in the medical management of ectopic tubal gestation, as the success rate is high in such cases. This is comparable to the study of Lipscomb et al. ${ }^{6}$

In the present study, the size of the ectopic mass selected was $<3.5 \mathrm{~cm} ; 24$ cases were in the group of $2-2.5 \mathrm{~cm}$ size. In a study conducted by Stovall TG et al7 in 1991, they treated 30 patients with unruptured ectopic pregnancy with sac size $\leq$ $3.5 \mathrm{~cm}$ with single dose Methotrexate and they found that 29 patients were treated successfully. This observation is comparable to present study.

23 out of 25 patients had pre-treatment beta-HCG level $<5000 \mathrm{IU} / \mathrm{L}$ and 2 had $5000 \mathrm{IU} / \mathrm{L}$, both cases were successfully treated with multi-dose Methotrexate. This is comparable with Lipscomb study. ${ }^{8}$ According to present study, success rate of medical management is high if the pretreatment beta-HCG level is low. Lipscomb et al 8 further stated that the initial level of beta-HCG is the best prognostic indicator for treatment success with Methotrexate.

In the present study $25(50 \%)$ out of 50 patients received single dose, of which 15 patients had beta-HCG < $1000 \mathrm{IU} / \mathrm{L}$ and 6 cases had $1000-2000$ IU/L and 4 cases with beta-HCG $>3000 \mathrm{IU} / \mathrm{L}$. These patients were followed up with serum beta-HCG levels and 23 (92\%) resolved within 14 days. So, single dose Methotrexate is effective in patients with initial low beta-HCG level. This is comparable to the study by Carla Merisio et al, ${ }^{9}$ Khaled B et al ${ }^{10}$ and Mamdoh E et al. ${ }^{11}$

\begin{tabular}{|c|c|c|}
\hline Beta-hCG Level & Lipscomb Study $^{8}$ & Present Study \\
\hline$>1000$ & $98.3 \%$ & $100 \%$ \\
\hline $1000-1999$ & $93 \%$ & $100 \%$ \\
\hline $2000-5000$ & $91.8 \%$ & $94 \%$ \\
\hline
\end{tabular}

Table 8. Comparison of Overall Success Rate of Present Study with Lipscomb according to Serum hCG Level

\begin{tabular}{|c|c|}
\hline Lipscomb et al $^{12}$ & $90 \%$ \\
\hline Banhart et al $^{13}$ & $89 \%$ \\
\hline Mamdoh Eskandar et al $^{11}$ & $94.3 \%$ \\
\hline Khalid B et Al$^{10}$ & $86.7 \%$ \\
\hline Carla Merisio et al & $90 \%$ \\
\hline Present Study & $94 \%$ \\
\hline \multicolumn{2}{|c|}{ Table 9. Comparison of Success } \\
Rate from Different Studies
\end{tabular}

\section{Summary}

The treatment of an ectopic pregnancy with methotrexate is safe and effective in carefully selected cases. Although, methotrexate treatment is beneficial, in that it allows one to avoid surgery in a patient; there are certain disadvantages. Medical treatment requires extended followup of patients, which can be cumbersome and difficult for some patients. The need to follow patients clinically until the serum HCG is undetectable and requires multiple visits, which takes 
valuable time from both patient and clinician. In comparison, surgical management is also safe, effective and often requires fewer followup visits. Furthermore, patients who are treated surgically have the same fertility rate as those who undergo methotrexate treatment. Some studies have even suggested that methotrexate doses used to treat ectopic pregnancy may worsen ovarian function in the short term, while others do not show an effect. These studies are limited and further studies are needed to evaluate the effect of low-dose methotrexate on ovarian reserve.

In conclusion, it is appropriate for clinicians to select suitable candidates for treatment of ectopic pregnancy with methotrexate. It is imperative to confirm diagnosis to avoid unnecessary administration of a chemotherapy, such as in a completed miscarriage or early intrauterine pregnancy that can have severe consequences for both mother and foetus.

There is usually no indication that methotrexate has to be administered at the first presentation of the patient, especially if she is clinically stable. If a woman is not stable or is in significant pain, she is not a candidate for medical management. Followup HCG or ultrasound can often avoid unnecessary or contraindicated administration of methotrexate. Moreover, patients should have appropriate counselling, willingness for followup and no absolute contraindications to methotrexate treatment. Despite high success rates, there remains a substantial rate of failure, particularly in low socioeconomic groups. The choice between two acceptable treatments, medicine or surgery, should be an informed and not reflective decision.

\section{CONCLUSION}

- Both medical therapy or conservative surgery should be viewed as appropriate first line therapies in many early unruptured ectopic pregnancies.

- Multiple-dose MTX treatment has a lower failure rate than single dose MTX. Single dose MTX is sufficient to treat persistent trophoblastic tissue after salpingostomy and ectopic pregnancies associated with low initial HCG values.

- Postoperative, prophylactic, single dose systemic MTX may reduce the incidence of persistent ectopic pregnancy after salpingostomy.

- Medical therapy appears more cost-effective than surgery, except when the initial HCG level is high and/or embryonic cardiac activity is observed.

\section{REFERENCES}

[1] Virkud A. Textbook of Modern obstetrics. Ectopic pregnancy. $2^{\text {nd }}$ edn. APC publishers, 2013:63-75.

[2] Cunninngham, Leveno, Bloom, et al. Textbook of Williams obstetrics-ectopic pregnancy. Chapter 19. McGraw Hill educations, 2014:pp 377.

[3] Bachman EA, Barnhart K. Medical management of ectopic pregnancy: a comparison of regimens. Clin Obstet Gynecol 2012;55(2):440-7.

[4] Medical Management of Ectopic Pregnancy. Clinical Guidelines. King Edward Memorial Hospital Section C Perth Western Australia DPMS Ref 8465, C 9.4.2.

[5] Menon S, Colins J, Barnhart KT. Establishing B-hcg cutoff to guide methotrexate treatment of ectopic pregnancy: a systematic review. Fertil Steril 2007;87(3):481-4.

[6] Lipscomb GH, Bran D, McCord ML, et al. Analysis of three hundred fifteen ectopic pregnancies treated with single-dose methotrexate. Am J Obstet Gynecol 1998;178(6):1354-8.

[7] Stovall TG, Ling FW, Gray LA. Single dose methotrexate for treatment of ectopic pregnancy. Obstet Gynecol 1991;77(5):754-7.

[8] Lipscomb GH, McCord ML, Stovall TG, et al. Predictors of success of methotrexate treatment in women with tubal ectopic pregnancies. $N$ Engl J Med 1999;341(26):1974-8.

[9] Merisio C, Anfuso S, Berretta R, et al. Single dose methotrexate for ectopic pregnancy treatment: preliminary data. Acta Bio Med 2005;76(1):33-6.

[10] Soliman KB, Saleh NM, Oman AA. Safety and efficacy of systemic methotrexate in the treatment of unruptured ectopic pregnancy. Saudi Med J 2006;27(7):1005-10.

[11] Mamdoh E. Single dose methotrexate for treatment of ectopic pregnancy: risk factors for treatment failure. Middle East Fertility Society Journal 2007;12(1):5762.

[12] Lipscomb GH, Givens VM, Meyer NL, et al. Comparison of multidose and single-dose methotrexate protocols for the treatment of ectopic pregnancy. Am J Obstet Gynecol 2005;192(6):1844-7.

[13] Barnhart KT, Gosman G, Ashby R, et al. The medical management of ectopic pregnancy: a meta-analysis comparing "single dose" and "multidose" regimens. Obstet Gynecol 2003;101(4):778-84. 